\title{
HELICON WAVE EXCITATION WITH HELICAL ANTENNAS
}

\author{
Max Light and Francis F. Chen \\ Electrical Engineering Department
}

PPG- 1520

October, 1994

Submitted to Physics of Plasmas. 


\title{
HELICON WAVE EXCITATION WITH HELICAL ANTENNAS \\ Max Light and Francis F. Chen \\ Electrical Engineering Department \\ University of California, Los Angeles, CA 90024-1594
}

\begin{abstract}
Components of the wave magnetic field in a helicon discharge have been measured with a single-turn, coaxial magnetic probe. Left- and right-handed helical antennas, as well as plane-polarized antennas, were used; and the results were compared with the field patterns computed for a non-uniform plasma. The results show that the right-hand circularly polarized mode is preferentially excited with all antennas, even those designed to excite the left-hand mode. For right-hand excitation, the radial amplitude profiles are in excellent agreement with computations.
\end{abstract}

PACS numbers: 52.35.Hr 52.70.Ds 52.40.Fd 52.80.Pi 


\section{I . INTRODUCTION}

Helicon waves in gaseous plasma were first observed by Lehane and Thonemann [1] and later studied extensively by Boswell [2], who pointed out that high density plasmas could be produced by helicon wave excitation with radiofrequency generators in the kilowatt regime. The reason for the high ionization efficiency of helicon discharges is not yet definitively known, though collisionless mechanisms, such as Landau and cyclotron damping, have been suggested by Chen [3] and Harvey and Lashmore-Davies [4], respectively. Helicon discharges have been suggested for semiconductor processing by Perry et al. [5], Nakano et al. [6], and Chen [7]; for gas laser excitation by Zhu and Boswell et al. [8]; for plasma accelerators by Chen [9]; and for plasma injection into toroidal confinement devices by Leowenhardt et al. [10].

To verify that helicon waves are indeed excited in rf discharges of this type, various workers have measured the wave fields with magnetic probes $[2,11]$. Comparison with theory, however, was inexact, since the theory was done for uniform plasmas whereas the plasmas had radially varying densities. Recently, Chen et al. [12] gave a method for computing the wave profiles for arbitrary density profiles with azimuthal symmetry, and this permitted the measurements in the present study to be compared with relevant theoretical curves.

Helicon waves are low-frequency whistler waves, which are well known to propagate with only right-hand circular polarization in free space. When bounded by a cylinder, however, these electromagnetic waves develop a large electrostatic component, and this allows them to have either right- or left-hand polarization and, therefore, plane polarization as well. Boswell et al. [2] have used straight antennas, which correspond to plane polarization; and Shoji et al. [11] have used left and right helical antennas, which correspond to circular polarization. In the data presented in Sec. III, we have compared all three types of antennas, with the unexpected result that right-hand polarization is preferentially excited by all of them. This discrepancy is discussed in Sec. IV. 


\section{II . SUMMARY OF THEORY}

Consider a cylindrical plasma with a radially varying density profile immersed in a coaxial static magnetic field $\mathbf{B}_{\mathbf{0}} \hat{\mathbf{z}}$. Let the first-order wave quantities vary as $\exp [\mathrm{i}(m \theta+k z-$ $\omega t)]$. Thus, an azimuthal mode number $m$ of +1 or -1 will correspond to a global right (+) or left (-) hand rotation of the wave pattern with respect to the static magnetic field. When the frequency $\omega$ lies far from both the ion and electron cyclotron frequencies, the ions motions can be neglected, and the electrons can be treated in the guiding center approximation. The only current in the plasma is then carried by the electron $\mathbf{E} \times \mathbf{B}$ drift:

$$
\mathbf{j}=-e n_{0}(r) \frac{\mathbf{E} \times \mathbf{B}_{\mathbf{0}}}{B_{0}^{2}}
$$

(the subscript on wave quantities has been suppressed). This current is used in Maxwell's equations

$$
\begin{aligned}
\nabla \cdot \mathbf{B} & =0 \\
\nabla \times \mathbf{E} & =i \omega \mathbf{B} \\
\nabla \times \mathbf{B} & =\mu_{0}\left(\mathbf{j}-i \omega \varepsilon_{0} \mathbf{E}\right)
\end{aligned}
$$

to give [12] the following dispersion relation for the $\hat{z}$ component of the wave magnetic field, $B_{z}:$

$$
B_{z}^{\prime \prime}+f(r) B_{z}^{\prime}+g(r) B_{z}=0
$$

with

$$
\begin{gathered}
f(r)=\frac{1}{r}-\frac{2 \alpha \alpha^{\prime}}{\beta}, \\
g(r)=\frac{\beta}{\gamma}-\frac{m^{2}}{r^{2}}-\frac{m}{k} \frac{\alpha^{\prime}}{\gamma r}\left(1+\frac{2 k^{2} \gamma^{2}}{\beta}\right)
\end{gathered}
$$

where $^{\prime}=\partial / \partial r$ and

$$
\alpha(r)=\frac{\omega}{k} \frac{\mu_{0} e}{B_{0}} n_{0}(r)
$$




$$
\gamma=1-\left(k_{0} / k\right)^{2}
$$

$$
\beta(r)=\alpha^{2}-k^{2} \gamma^{2} .
$$

The other components of the wave magnetic field are given by [12]

$$
\begin{gathered}
\beta B_{r}=\frac{i m}{r} \alpha B_{z}+i k \gamma B_{z}^{\prime} \\
\beta B_{\theta}=-\alpha B_{z}^{\prime}-\frac{m}{r} k \gamma B_{z} .
\end{gathered}
$$

Let $p(r)$ be the normalized density profile and $\alpha_{0}$ the maximum value of $\alpha$ (at $r=0$ ), so that

$$
\alpha(r)=\alpha_{0} p(r) .
$$

Eq. (5) is integrated numerically for given $p(r)$, and the eigenvalue $\alpha_{0}$ is adjusted until the boundary condition ${ }^{12} B_{r}=0$ is satisfied, where $\mathrm{B}_{\mathrm{r}}$ is given in terms of $\mathrm{B}_{\mathrm{z}}$ by Eq. (11). The profile factor $p(r)$ can be an analytic function or a polynomial fit to experimental data. This procedure requires that the parallel wavenumber $k$ be known; in this paper we have assumed it to be twice the antenna length.

Figure 1 shows examples of the radial profiles of $B_{r}, B_{\theta}$, and $B_{z}$; and Fig. 2 shows the field line patterns in a cross-sectional plane, computed for a parabolic density profile. Since the wave quantities vary as

$$
B \propto f(r) e^{i(m \theta+k z-\omega t)},
$$

the patterns for $m= \pm 1$ remain unchanged in time or space, and simply rotate in the $\pm \theta$ direction, respectively, in time at a fixed position $z$, and in the direction as $z$ increases at a given time. The symmetric $m=0$ mode, on the other hand, changes its field pattern so that the wave 
Fig. 1. Radial profiles of the wave magnetic field components for the (a) $\mathrm{m}=+1$, (b) $\mathrm{m}=$ 1 , and (c) $m=0$ modes for a parabolic density profile $\mathrm{n}_{0}=1-(\mathrm{r} / \mathrm{a})^{2}$.

electric field goes from purely electromagnetic to purely electrostatic in every half wavelength [3].

Fig. 2. Patterns of the B-field lines (heavy) and E-field lines (light) in the $x-y$ plane for the (a) $\mathrm{m}=+1$ and (b) $\mathrm{m}=-1$ modes for a parabolic density profile. 


\section{EXPERIMENTAL ARRANGEMENT}

The experimental apparatus is shown in Fig. 3. Plasma density was measured with ion saturation current to Langmuir probes at all of the radial access flanges. The probes were calibrated against microwave interferometry. Table I lists the relevant parameters. The antenna is located near one end of the machine. Though it launches waves in both directions, we assume that the parallel wave vector $\mathbf{k}$ of the waves under study points toward the midplane of the vacuum chamber.

Fig. 3. Experimental apparatus.

TABLE I. Experimental parameters

\begin{tabular}{|l|l|}
\hline Gas & Argon \\
\hline Base pressure & $2-5 \times 10^{-5}$ Torr \\
\hline Fill pressure & $8 \mathrm{mTorr}$ \\
\hline Transmitted power & $1.9 \mathrm{~kW}(<10 \%$ reflection $)$ \\
\hline Excitation frequency & $27.12 \mathrm{MHz}$ \\
\hline Magnetic field & $800 \mathrm{G}$ \\
\hline Length of rf pulse & $150 \mathrm{msec}$ \\
\hline
\end{tabular}


Three different types of antennas were used in this experiment, as shown in Fig. 4. The first is the well known [13] Nagoya Type III antenna, which has $m= \pm 1$ symmetry and plane polarization. The other two are also Type III antennas but have $180^{\circ}$ helical twists instead of the straight horizontal legs in the original Nagoya antenna. If the helicity is such that the horizontal legs twist in the counterclockwise direction when the observer moves in the direction of $\mathbf{k}$, as defined above, the antenna is called an R-antenna. Conversely, if the legs twist in the clockwise direction along $\mathbf{k}$, the antenna is an L-antenna. The untwisted configuration will be called simply the Nagoya, or $\mathrm{N}$-antenna. The axial lengths are $15.2,16$, and $13.3 \mathrm{~cm}$ for the $\mathrm{R}, \mathrm{L}$, and $\mathrm{N}$ antennas, respectively. They are wrapped around the quartz tube external to the plasma.

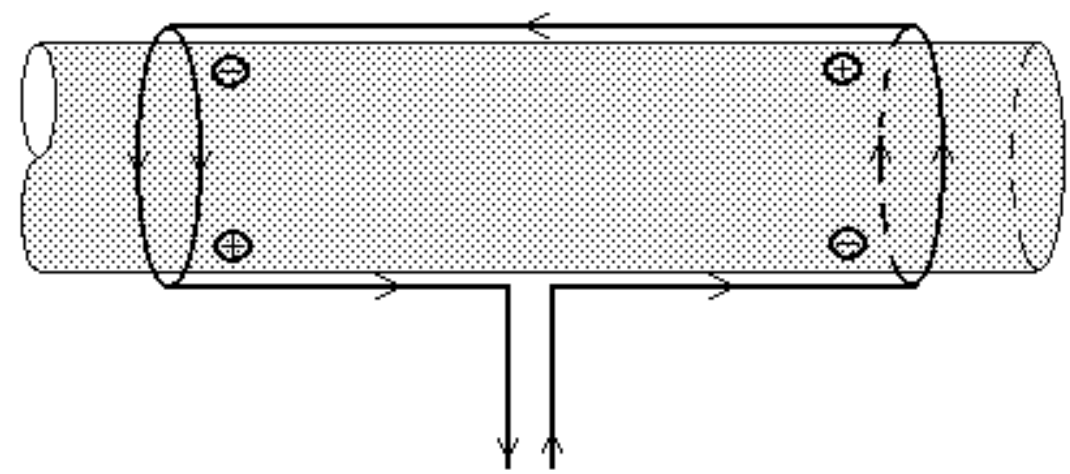

Fig. 4a. Nagoya Type III plane-polarized antenna.

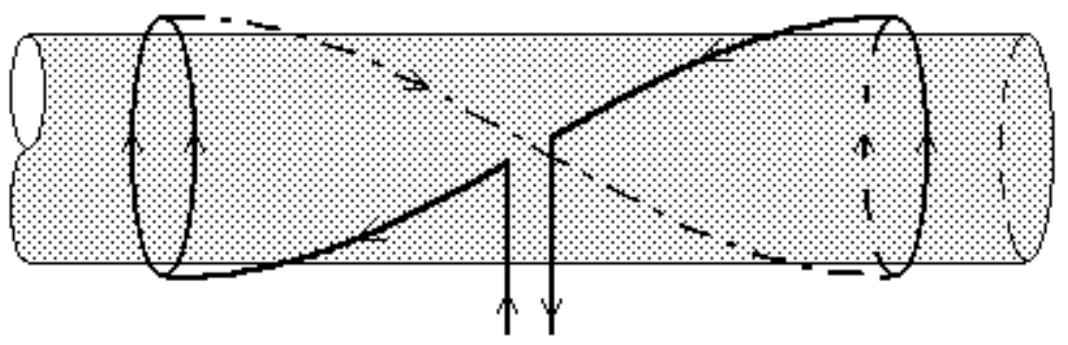

Fig. 4b. R-type antenna. $\mathbf{B}_{\mathbf{0}}$ and $\mathbf{k}$ are from right to left.

Nagoya Type III antennas are especially effective because they generate space charges which give rise to an internal electrostatic field in the plasma. The mechanism has been described by Chen [14] and will be given in more detail here. Consider the classical Nagoya Type III antenna structure of Fig. 5. 


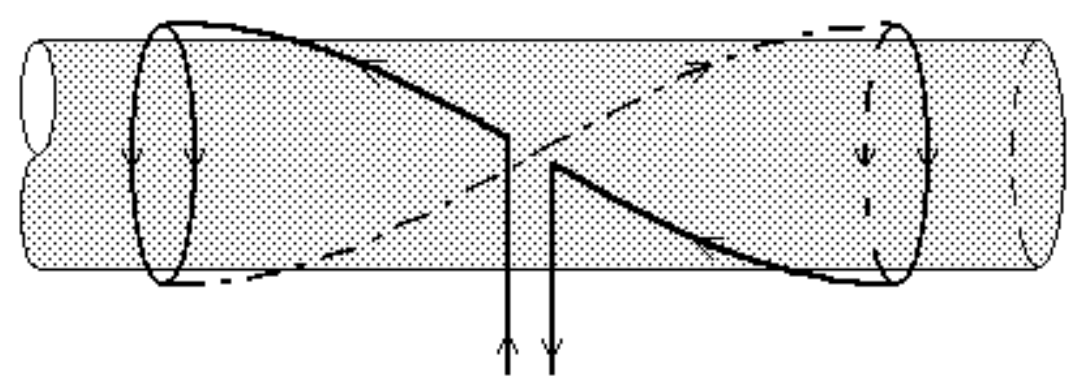

Fig. 4c. L-type antenna. $\mathbf{B}_{\mathbf{0}}$ and $\mathbf{k}$ are from right to left.

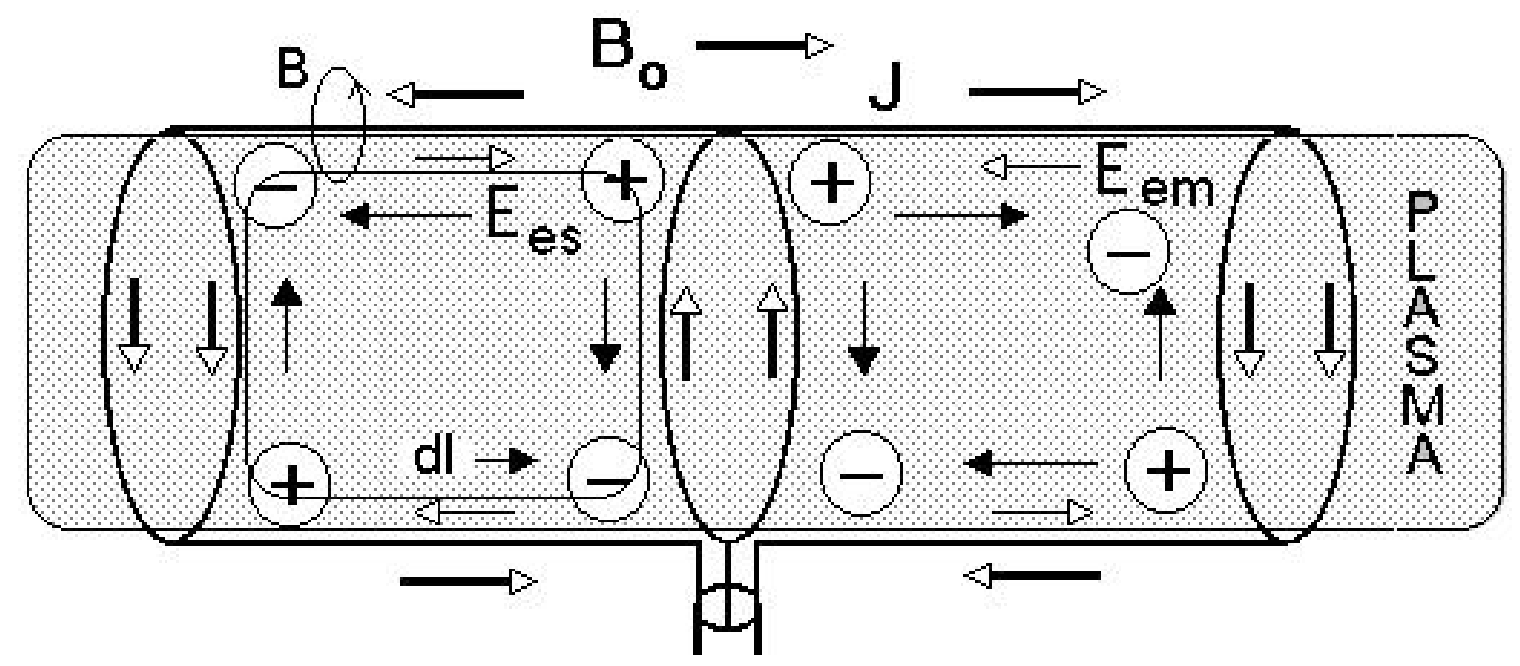

Fig. 5. Mode coupling mechanism for the N-antenna.

During the half cycle when it is increasing, the rf current will flow in the various parts of the antenna as shown. We may assume that the current magnitude is constant along any leg of the antenna because the free space wavelength at the frequencies of interest is much larger than the antenna length. The current on the horizontal legs will induce an rf magnetic field, which will in turn induce an electromagnetic electric field given by

$$
\oint \mathbf{E} \cdot d \mathbf{l}=-\iint_{S} \frac{\partial}{\partial t}(\mathbf{B} \cdot d \mathbf{s}),
$$

where the integral is taken along the path shown. This electromagnetic electric field will cause electrons to flow along the dc magnetic field as long as the plasma is a good conductor in the parallel direction. Since the antenna is either periodic or finite in the $z$ direction, the electron motion will set up a space charge on each field line until the electrostatic field of the 
space charge cancels the induced electromagnetic electric field in the parallel direction, so that the net $E_{z}$ is nearly zero, as required in a good conductor. The space charge distribution also gives rise to a perpendicular electrostatic field, which in this geometry is in the same directon as the electromagnetic electric field, and thus enhances it. The enhancement factor is of order $\left(k_{\perp} / k_{z}\right)^{2}$. This $\mathrm{E}_{\perp}$-field is not shielded out by the plasma by the skin effect because the electrons cannot move across $\mathbf{B}_{\mathbf{0}}$ in the direction of $\mathbf{E}_{\perp}$.

From Figs. $2 \mathrm{a}$ and $2 \mathrm{~b}$ we see that the electric field lines of the wave do not close on themselves. Thus, the wave creates space charges which give rise to a more or less planar electrostatic field near the axis. The overlap between this field pattern and that created by the antenna is the coupling mechanism. The plane $\mathrm{N}$-antenna shown in Fig. 3 will generate an Efield pattern that is plane-polarized; that is, a linear combination of the $m=+1$ and $m=-1$ modes. It can, therefore, excite either mode, but not an $m=0$ mode. By introducing a twist to the horizontal legs of the $\mathrm{N}$-antenna, we would induce in the plasma an electrostatic field which rotates in space, matching the instantaneous mode pattern of the $m=+1$ or $m=-1$ mode. The R-antenna would couple to an $m=+1$ mode propagating toward the far end of the machine and an $m=-1$ mode propagating toward the near end (which, we assume, is absorbed). The L-antenna would do the reverse. Note, however, that the antenna patterns rotate only in space, not in time, and are therefore not perfect matches to the $m= \pm 1$ mode patterns. Rotating antenna fields can be generated with bifilar, phase shifted antennas, but these were not used in this work.

The direction of rotation for the $\mathrm{R}(m=+1)$ and $\mathrm{L}(m=-1)$ helicon waves refers to the direction of the dc magnetic field $\mathbf{B}_{\mathbf{0}}$, whereas the helicity of the $\mathrm{R}$ and $\mathrm{L}$ antennas refers to the direction of $\mathbf{k}$. Thus, by reversing the direction of $\mathbf{B}_{\mathbf{0}}$, we can excite an $\mathrm{R}$ wave with the $\mathrm{L}$ antenna or an $\mathrm{L}$ wave with the $\mathrm{R}$ antenna. We have explored all four cases and denote them as follows. When $\mathbf{B}_{\mathbf{0}}$ and $\mathbf{k}$ are in the same direction, the $\mathrm{R}$ and $\mathrm{L}$ antennas generate cases $(\mathrm{R} \|)$ and $(\mathrm{L} \|)$, respectively. When $\mathbf{B}_{\mathbf{0}}$ and $\mathbf{k}$ are in opposite directions, the $\mathrm{R}$ and $\mathrm{L}$ antennas generate cases ( $\mathrm{R \# )}$ and $\mathrm{R}(\#)$, respectively. Thus, the $\mathrm{R}$ helicon wave would be expected to be excited in either the $(\mathrm{R} \|)$ or $(\mathrm{L} \#)$ case, and the $\mathrm{L}$ helicon wave in the $(\mathrm{R \# )}$ or $(\mathrm{L} \|)$ case. 
The single-turn magnetic pickup loop used to measure the relative B-fields of the wave is shown in Fig. 6. The $\theta$ and $z$ components were measured with the same probe by rotating it $90^{\circ}$ with respect to $\mathbf{B}_{\mathbf{0}}$. The $r$ component was measured with the pickup loop bent back $90^{\circ}$ from that shown in Fig. 6. Figure 7 shows the configuration for measuring the relative phases of the components of the wave fields. All profile and relative phase measurements were performed at port 1. Signals from the probes were recorded on a Hewlett Packard 54510A digital oscilloscope at a real-time rate of $1 \mathrm{GHz}$. Digital data from the oscilloscope were stored on an Apple Macintosh IIci computer using LabView 2.2.1 software. Magnetic probe signals were measured over a 200-nS window occurring at the same time during each rf pulse. Amplitude data were averaged over 10 shots.

Fig. 6. Single-turn magnetic probe.

Fig. 7. Configuration for measurements of the relative phases of the wave components.

The parameter study consisted of measuring the radial profiles and relative phases of $B_{r}, B_{\theta}$, and $B_{z}$ at port 1, and the radial density profiles at each port. This was repeated for each of three antennas with the magnetic field parallel and antiparallel to $\mathbf{k}$.

\section{EXPERIMENTAL RESULTS}

\section{Density Profiles}


Plasma density radial profiles were measured at all three radial access flanges. Figure 8 shows that each configuration assigned to excite a particular mode, or mode mix in the case of the Nagoya antenna, gives a distinct radial and axial profile. For instance, the $m=+1$ mode configurations ( $\mathrm{R} \|$ and $\mathrm{L \#}$ ) give identical profiles, and the $\mathrm{m}=-1$ configurations (L\| and $\mathrm{R \#}$ ) give identical ones which are distinct from the $m=+1$ mode configurations. In addition, the Nagoya antenna gives identical density profiles regardless of the direction of $\mathbf{B}_{\mathbf{o}}$ relative to $\mathbf{k}$. The $\mathrm{R} \|$ and L\# configurations give the best axial sustainment of the discharge and have the highest peak density at port 2 , where the gas is fed into the chamber. The Nagoya antenna configurations give equal radial profiles at ports 1 and 2, with a large drop in density from ports 2 to 3. L\| and R\# configurations have the worst axial characteristics with a large drop in density from ports 1 to 2 and 2 to 3 .

While no real conclusions from these density measurements can be made about the exact mode content (the configurations were set up to attempt excitation of particular modes), it is clear that identical discharges are created, as expected, if both the antenna helicity and the $\mathbf{B}_{0}$ direction are reversed.

\section{Field Profiles}

Figures 9 to 14 give the radial profiles of $B_{r}, B_{\theta}$, and $B_{z}$, plotted against theoretical curves for the $m=+1$ and -1 modes. The theoretical wave component profiles were computed from Eq. (5) using a polynomial fit to the measured radial density profile in each case. Only the absolute values of $B_{j}$ are shown, since the magnetic probes were set up to measure amplitude only, not phase. Zero crossings will be dealt with in the next section. The meassured wave profiles were assumed to be a linear superposition of the $m=+1,-1$ and 0 modes. 
Fig. 8. Radial profiles of plasma density for each excitation configuration. (a) $R \|$ and (b) L\# are designed to excite $m=+1$. (c) $L \|$ and (d) R\# are designed to excite $m=-1$. (e) $N \|$ and (f) N\# should excite both modes. 
Fig. 9

Fig. 10

Fig. 11

Fig 12

Fig. 13

Fig. 14

Normalized radial profiles for the R\|, L\#, and L\|, R\#, N\|, and N\# configurations, respectively. Points: data; solid lines: $\mathrm{m}=+1$ theory; dashed lines: $\mathrm{m}=-1$ theory. 
To investigate the relative content of each mode, normalized $B_{r}, B_{\theta}, B_{z}$ profiles for $\mathrm{m}=$ $+1,-1$, and 0 were calculated for each measured density profile. A re-normalized linear combination of these profiles was then fit to the normalized data. This can be written as

$$
C_{+} B_{z+}(r)+C_{-} B_{z-}(r)+C_{0} B_{z 0}(r)=B_{z \text { meas }}(r)
$$

where the C's were solved to make the best fit. The results are shown in Table II. Figures 9-14 and Table II clearly show that the $\mathrm{m}=+1$ mode is dominant for all excitation configurations. There is little trace of the $\mathrm{m}=0$ mode, since we see from the theoretical $\mathrm{m}=0$ curves of Fig. 1 that $B_{\theta}(\mathrm{r})$ starts and ends at zero with no zero crossing, while $B_{r}(\mathrm{r})$ has a minimum at $\mathrm{r}=0$; and none of the measurements show these features. The only configurations showing a strong deviation from the $m=+1$ profiles are $L \|$ and $R \#$, which are designed to excite the $m=-1$ mode. The experimental data for the $\mathrm{L} \|$ and $\mathrm{R} \#$ configurations show a large peak in the amplitude of $B_{\theta}(\mathrm{r})$ near the outside edge of the plasma, a feature not predicted for any mode, and not yet explained. On the other hand, the $B_{\theta}(\mathrm{r})$ measurements for $\mathrm{L} \|$ and R\# have zero crossings agreeing with the $\mathrm{m}=+1$ mode, and $B_{r}(\mathrm{r})$ also agrees more closely with the $\mathrm{m}=+1$ than with the $\mathrm{m}=-1$ mode profile.

TABLE II. Calculated mode content from least squares fit to $B_{z}$ profile data.

\begin{tabular}{c|ccc}
\hline Excit. Config. & $\mathrm{C}_{-1}$ & $\mathrm{C}_{0}$ & $\mathrm{C}_{+1}$ \\
\hline $\mathrm{R} \|$ & 0.07 & 0.00 & 0.93 \\
$\mathrm{R} \#$ & 0.00 & 0.11 & 0.89 \\
$\mathrm{~L} \|$ & 0.00 & 0.29 & 0.71 \\
$\mathrm{~L} \#$ & 0.00 & 0.00 & 1.00 \\
$\mathrm{~N} \|$ & 0.00 & 0.15 & 0.85 \\
$\mathrm{~N} \#$ & 0.00 & 0.11 & 0.89 \\
\hline
\end{tabular}




\section{Phase Measurements}

To verify that the phase of $B_{\theta}(\mathrm{r})$ actually changes where its amplitude goes to zero, we have measured the change of phase of each $\mathbf{B}$ component with radius, relative to the phase of the signal on a fixed magnetic probe at port 1 . The moveable probe was placed at the same port, $90^{\circ}$ away in azimuth. Either probe can be set to measure any component of $\mathbf{B}$. Since the orientation of the $B_{\theta}$ and $B_{z}$ probes was not recorded each time they were inserted, there is an ambiguity of $180^{\circ}$ in the phase of these signals, depending on the orientation of the loop. The relative phase measurement given in Figs. 15-17 should be interpreted with this ambiguity in mind. The vertical line in each figure corresponds to the point where the theoretically calculated $B_{\theta}$ crosses zero for the $\mathrm{m}=+1$ mode.

All excitation configurations except R\# show phase discontinuities in $B_{\theta}(\mathrm{r})$ in good agreement with the theoretically predicted zero crossings for the $\mathrm{m}=+1$ mode. Note that $\Phi_{\mathrm{zz}}$ and $\Phi_{\theta z}$ for both $\mathrm{m}=+1$ mode excitation configurations give an initial relative phase about 270 degrees different from the rest of the data points for each phase profile (Fig. 15). We believe that this is an artifact of alignment. In each case, the moveable probe was rotated to measure $B_{z}$. Near $\mathrm{r}=0$, however, $B_{\theta}$ is much larger than $B_{z}$, and these probes could have picked up a $B_{\theta}$ signal if they were not perfectly aligned. Phase data for the R\# configuration does not show evidence for a zero crossing. This is in conflict with the wave amplitude data for this configuration, which show a dip in $\left|B_{\theta}\right|$ at the proper radius for the $\mathrm{m}=+1$ zero crossing.

Relative phase differences between components whose phases should remain constant with radius for an $\mathrm{m}=+1$ mode $\left(\Phi_{\mathrm{rz}}, \Phi_{\mathrm{zz}}, \Phi_{\mathrm{r} \theta}\right.$, and $\left.\Phi_{\theta \mathrm{z}}\right)$ do indeed stay constant for all excitation configurations. Figure $1 \mathrm{~b}$ shows that $B_{z}$ should also experience a zero crossing for the $\mathrm{m}=-1$ mode, i.e., $\Phi_{\mathrm{zz}}$ and $\Phi_{\theta \mathrm{z}}$ should experience a discontinuity near the edge of the plasma. This was not experimentally observed.

Identification of the modes so far has been based on the radial profiles of $\mathbf{B}$ alone. To 
check that the mode patterns actually rotate in the right-hand direction when the profiles agree with the $\mathrm{m}=+1$ predictions, we have measured the phase of $B_{\theta}$ on two probes spaced $90^{\circ}$ apart in azimuth. The results for the six configurations are shown in Table III. The two configurations designed to excite the $\mathrm{m}=-1$ mode--namely, $\mathrm{R} \#$ and $\mathrm{L} \|$--also gave patterns rotating in the right-hand direction, in agreement with the radial profiles.

Fig. 15

Fig. 16

Fig. 17

Figs. 15-17. Relative phase measurements for the $\mathrm{m}=+1, \mathrm{~m}=-1$, and $\mathrm{m}= \pm 1$ (Nagoya) excitation configurations, respectively. The vertical line corresponds to the theoretical $\mathrm{m}=+1 B_{\theta}$ zero-crossing .

TABLE III. Rotation measurement results

\begin{tabular}{c|cc}
\hline Excit. Config. & Phase (degrees) & Rotation Dir. \\
\hline $\mathrm{R} \|$ & 93.7 & Right \\
$\mathrm{L} \#$ & 97.6 & Right \\
$\mathrm{N} \|$ & 124.9 & Right \\
$\mathrm{N} \#$ & 82.0 & Right \\
$\mathrm{R} \#$ & 82.0 & Right \\
L\| & 78.0 & Right \\
\hline
\end{tabular}




\section{Discussion}

The wave amplitude and phase measurements show that the $\mathrm{m}=+1$ mode appears to dominate for all excitation configurations with different antennas and magnetic field directions. Those configurations designed to excite this mode give wave patterns in good agreement with theory. On the other hand, exciting the $\mathrm{m}=-1$ mode does not appear to be possible even with the configurations designed to excite this mode. The $m=0$ mode shows up only as a small mixed component in the statistical fit results of Table II.

We can use two arguments to explain these results. First, examine the parameter $\alpha_{0}$ from Eqs. (8) and (13),

$$
\alpha_{0}=\frac{\omega}{k} \frac{\mu_{0} e n_{0}}{B_{0}}
$$

which corresponds to the value of $\alpha$ at the $r=0$ density maximum. Assuming $k$ is set by the antenna, and that the antenna length corresponds to a half wavelength in the plasma, we can rewrite this as

$$
n_{0}=\frac{\alpha_{0} k B_{0}}{\omega e \mu_{0}}
$$

From this, the expected peak density for each mode can be calculated using the experimentally measured density profiles at port 1 :

$$
\begin{aligned}
& \mathrm{n}_{0}(+1)=6.4-6.8 \times 10^{12} \mathrm{~cm}^{-3} \\
& \mathrm{n}_{0}(-1)=3.6-4.9 \times 10^{13} \mathrm{~cm}^{-3}
\end{aligned}
$$

The measured peak density at port 1 ranged from $6.2-8.8 \times 10^{12} \mathrm{~cm}^{-3}$, in agreement with the $\mathrm{m}=+1$ requirement. The $\mathrm{rf}$ power may have been insufficient to produce the density required for the $\mathrm{m}=-1$ mode. In Eq. (18) there is an uncertainty in the value of $k$, since that was not measured directly. If we allow a 50\% decrease in the parallel wavelength, $\mathrm{n}_{0}(+1)$ and $\mathrm{n}_{0}(-1)$ will increase by factors of 2.8 and 1.5 , respectively, for a given density profile. A $50 \%$ in- 
crease in the parallel wavelength will decrease $\mathrm{n}_{0}(+1)$ and $\mathrm{n}_{0}(-1)$ by factors of 2.0 and 1.2 , respectively. Thus, our hypothesis is not affected by variations of this order.

Second, in the wave field patterns of Figs. $2 a$ and $2 b$, one notices that the $m=+1$ mode gives a larger area of straight electrostatic field lines for the antenna to couple into as compared to the $\mathrm{m}=-1$ mode pattern. Thus, from the standpoint of antenna coupling, the $\mathrm{m}=$ +1 mode again seems easier to excite.

Since the straight $\mathrm{N}$-antenna can couple to either the $\mathrm{m}=+1$ or the $\mathrm{m}=-1$ mode, it is reasonable that it should excite only the mode whose dispersion relation requires a density that is easily achieved with the rf power available. The $\mathrm{L}$ antenna, however, has a helicity which does not match at all that of the +1 mode; yet it manages to couple to it. To understand this, we note that the half-wavelength antennas which were used necessarily excite a broad spectrum of k-numbers. Consider first the $\mathrm{N}$-antenna. Fig. 18 shows the juxtaposition of two half-wavelength $\mathrm{N}$-antennas to form a full-wavelength antenna. Only the horizontal legs of the antenna are necessary for inducing the electric field and space charge pattern shown for the half-cycle when the antenna current is increasing. The end-rings are needed only for closing the circuit. Fortuitously, the E-field induced by the end-ring currents is in the same direction as the perpendicular space charge field, and therefore the end-rings affect the antenna coupling only in a beneficial sense.

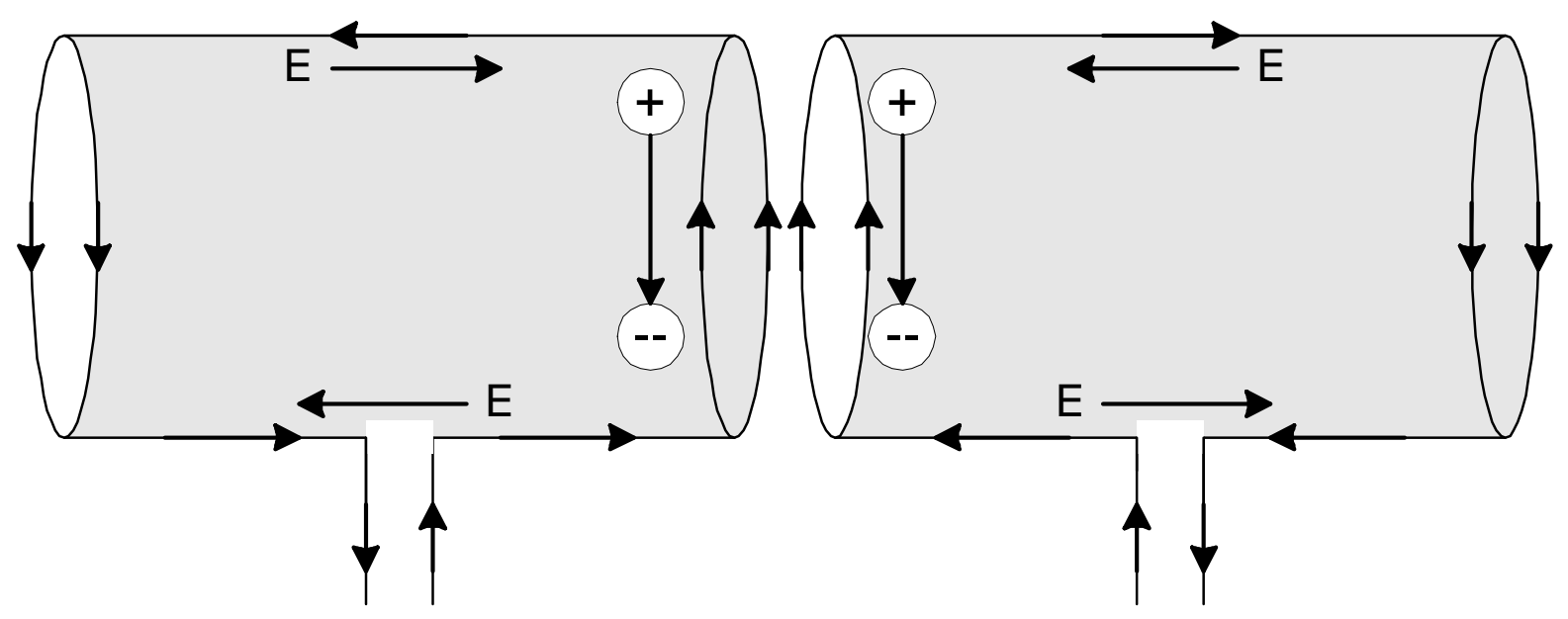

Fig. 18. Two half-wavelength $\mathrm{N}$-antennas phased so as to create a full-wavelength antenna. 
This is not true of helical antennas. Fig. 19a shows an ideal full-wavelength helical antenna composed of two half-wavelength helices. The two halves can be joined at the midplane; and since the ring currents there cancel each other, those rings can be omitted altogether, as well as the second current feed. The electric field and space charge distribution shown in Fig. 19a applies only to the field lines at the top and bottom of the cylinder; it is understood that this pattern has helical symmetry. The parallel wavelength is still twice the length of each section. When only one half-wavelength antenna (the one on the left) is used, however, the ring current at the right end is not cancelled, and this current induces an E-field as if there had been another section on the right with opposite polarity to that in Fig. 19a, as shown in Fig. 19b. The E-field and space charge configuration of this combination has a parallel wavelength equal to the length of one of the sections alone, and therefore half the wavelength excited by an $\mathrm{N}$-antenna of equivalent length. An $\mathrm{m}=-1$ mode with this value of $k$ would require a peak density of order $7 \times 10^{13} \mathrm{~cm}^{-3}$, well beyond the capability of our equipment at $800 \mathrm{G}$. It is possible that the broad spectrum of an L-antenna with uncompensated end-ring currents could include an an $\mathrm{m}=+1$ component, since these currents in a helical antenna apparently affect the antenna pattern much more than in an $\mathrm{N}$-antenna. Finally, there is a possibility that the L-antenna excites an $\mathrm{m}=+1$ propagating toward the pump end of the chamber, which is then reflected into the main chamber. If the coupling to the $m=-1$ mode is extremely weak, this small reflected $\mathrm{m}=+1$ component may be the dominant one that is observed.

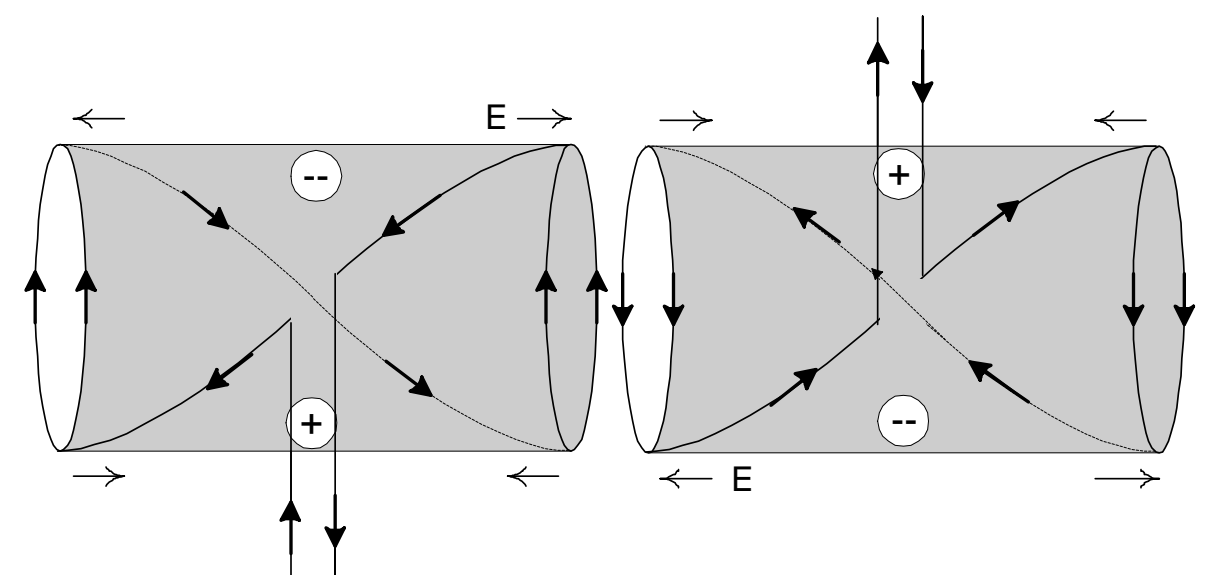

Fig. 19a. Two half-wavelength helical antennas phased to create a full-wavelength antenna. 


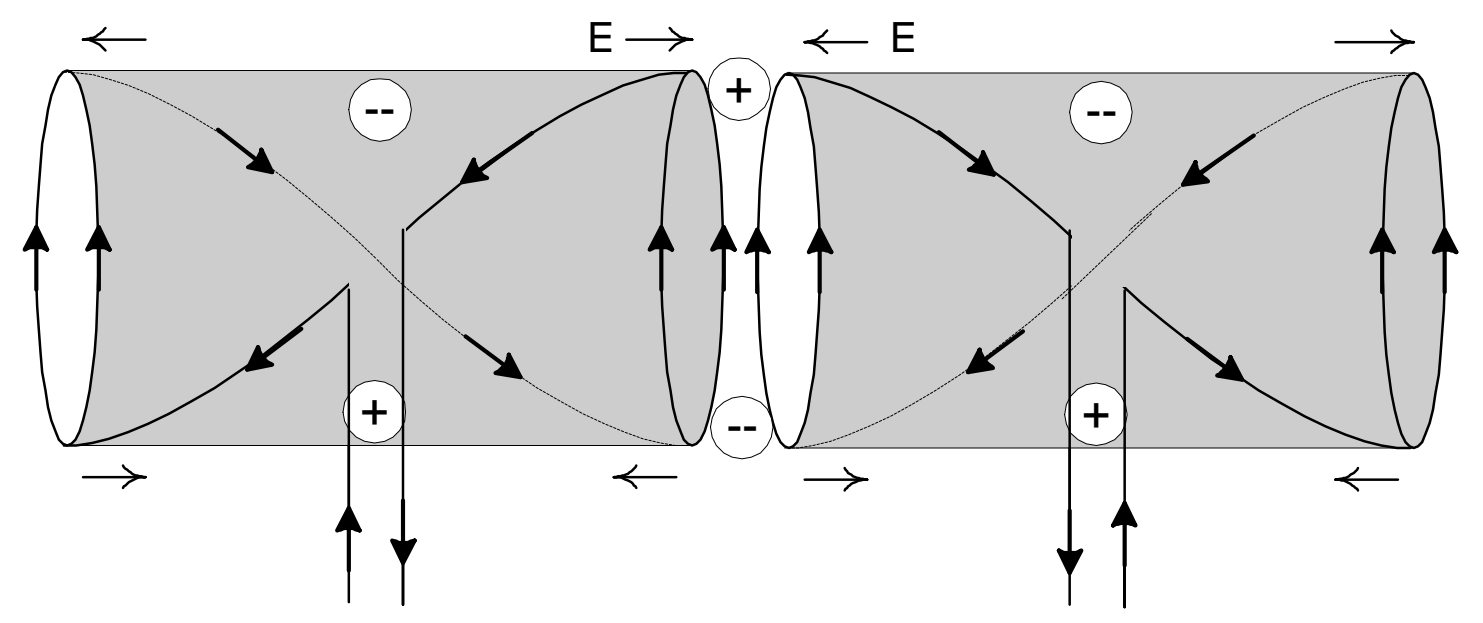

Fig. 19b. Two half-wavelength helical antennas phased to generate wavelengths half as long.

In conclusion, helicon wave magnetic field patterns for a cold, collisionless plasma with a radial density gradient have been numerically solved and compared to experimental measurements for different antenna and static magnetic field combinations. The $\mathrm{m}=+1$ mode dominates for all excitation configurations. Left-hand polarized $(m=-1)$ modes or mode mixtures containing an $\mathrm{m}=-1$ component are not observed for any configuration. We believe that the reason is that the $m=-1$ dispersion relation requires higher peak density than was attainable, and that the antenna designs used were less than ideal for exciting pure circularly polarized modes.

\section{ACKNOWLEDGMENTS}

This work was supported by the Semiconductor Research Corp., the Wisconsin Engineering Research Center for Plasma-Aided Manufacturing, the Lawrence Livermore Laboratory Plasma Physics Research Institute, and National Science Foundation Equipment Grant ECS 92-12432. 


\section{FIGURE CAPTIONS}

Fig. 1. Radial profiles of the wave magnetic field components for the (a) $m=+1$, (b) $m=-1$, and (c) $\mathrm{m}=0$ modes for a parabolic density profile $\mathrm{n}_{0}=1-(\mathrm{r} / \mathrm{a})^{2}$.

Fig. 2. Patterns of the B-field lines (solid) and E-field lines (dashed) in the $x-y$ plane for the (a) $\quad \mathrm{m}=+1$ and (b) $\mathrm{m}=-1$ modes for a parabolic density profile.

Fig. 3. Experimental apparatus.

Fig. 4. Antenna types used in the experiment: (a) Nagoya Type III, (b) R, and (c) L. B and $\mathbf{k}$ are assumed to point from right to left.

Fig. 5. Mode coupling mechanism for the N-antenna.

Fig. 6. Single-turn magnetic probe.

Fig. 7. Configuration for measurements of the relative phases of the wave components.

Fig. 8. Radial profiles of plasma density for each excitation configuration. (a) $R \|$ and (b) L\# are designed to excite $m=+1$. (c) $\mathrm{L} \|$ and (d) R\# are designed to excite $m=-1$. (e) $\mathrm{N} \|$ and (f) N\# should excite both modes.

Fig. 9. Normalized radial profiles for the $\mathrm{R} \|$ configuration. Points: data; solid lines: $\mathrm{m}=+1$ theory; dashed lines: $\mathrm{m}=-1$ theory.

Fig. 10. Normalized radial profiles for the L\# configuration. Points: data; solid lines: $\mathrm{m}=+1$ theory; dashed lines: $\mathrm{m}=-1$ theory.

Fig. 11. Normalized radial profiles for the $\mathrm{L} \|$ configuration. Points: data; solid lines: $\mathrm{m}=+1$ theory; dashed lines: $\mathrm{m}=-1$ theory.

Fig. 12. Normalized radial profiles for the R\# configuration. Points: data; solid lines: $\mathrm{m}=+1$ theory; dashed lines: $\mathrm{m}=-1$ theory.

Fig. 13. Normalized radial profiles for the $\mathrm{N} \|$ configuration. Points: data; solid lines: $\mathrm{m}=+1$ theory; dashed lines: $\mathrm{m}=-1$ theory.

Fig. 14. Normalized radial profiles for the N\# configuration. Points: data; solid lines: $\mathrm{m}=+1$ theory; dashed lines: $\mathrm{m}=-1$ theory.

Fig. 15. Relative phase measurements for the $\mathrm{m}=+1$ excitation configurations. The vertical line corresponds to the theoretical $\mathrm{m}=+1 B_{\theta}$ zero-crossing .

Fig. 16. Relative phase measurements for the $\mathrm{m}=-1$ excitation configurations. The vertical line corresponds to the theoretical $\mathrm{m}=+1 B_{\theta}$ zero-crossing . 
Fig. 17. Relative phase measurements for the mode mix excitation configurations. The vertical line corresponds to the theoretical $\mathrm{m}=+1 B_{\theta}$ zero-crossing .

Fig. 18. Two half-wavelength $\mathrm{N}$-antennas phased so as to create a full-wavelength antenna.

Fig. 19. Two half-wavelength helical antennas phased to create (a) an ideal full-wavelength antenna and (b) an antenna exciting half the intended wavelength. 


\section{REFERENCES}

1. Lehane, J.A. and Thonemann, P.C., Proc. Phys. Soc. 85, 301 (1965).

2. Boswell, R.W., Plasma Phys. and Controlled Fusion 26: 1147 (1984).

3. Chen, F.F., Plasma Phys. and Controlled Fusion 33: 339 (1991).

4. Harvey, B.M. and Lashmore-Davies, C.N., Phys. Fluids B 5: 3864 (1993).

5. Perry, A.J., Vender, D., and Boswell, R.W., J. Vac. Sci. Technol. B 9: 310 (1991).

6. Nakano, T., Gottscho, R.A., Sadeghi, N., Trevor, D.J., Boswell., R.W., Perry, A.J., Lee, T.C., Giapis, K.P., and Margot, J., Oyo Buturi (Japan Soc. of Appl. Phys.) 61: 711 (1992).

7. Chen, F.F., Helicon Plasma Sources, in High Density Plasma Sources, ed. by Oleg A. Popov (Noyes Publications), to be published (1994).

8. Boswell, R.W., Porteus, R.K., Prytz, A., Bouchoule, A., and Ranson, P. Phys. Lett. A 91: 163 (1982).

9. Chen, F.F., Physica Scripta T30: 14 (1990).

10. Loewenhardt, P.K., Blackwell, B.D., Boswell, R.W., Conway, G.D., and Hamberger, S.M., Phys. Rev. Lett. 67: 2792 (1991).

11. Shoji, T., Sakawa, Y., Nakazawa, S., Kadota, K, and Sato, T., Plasma Sources Sci. Technol. 2: 5 (1993).

12. Chen, F.F., Hsieh, M.J., and Light, M., Plasma Sources Sci. Technol. 3: 49 (1994).

13. Okamura, S., Adati, K., Aoki, T., Baker, D.R., Fujita, H., Garner, H.R., Hattori, K., Hidekuma, S., Kawamoto, T., Kumazawa, R., Okubo, Y., and Sato, T., Nuclear Fusion 26: 1491 (1986).

14. Chen, F.F. and Chevalier, G., J. Vac. Sci. Technol. A 10: 1389 (1992). 